\title{
La pollution des eaux pluviales urbaines en réseau d'assainissement unitaire - Caractéristiques et origines
}

\author{
Urban wet weather pollution in combined \\ sewer networks - Characteristics and origins \\ par M.C. Gromaire-Mertz* \\ CEREVE (ENPC, ENGREF, UPVM)
}

This research aimed to fit out an experimental urban catchment area (Le Marais catchment in Paris), provided with a combined sewer network and situated downtown, so as to obtain a description of the pollution of urban wet weather flows at different levels of the urban water cycle.

This approach allowed:

- A detailed characterisation of the pollution of waste water, of urban runoff from roofs, streets and yards and of combined flow at the catchment outlet. The parameters we focused on were:

- event mean concentrations of SS, VSS, COD, BODS, heavy metals $(C d, C u, P b, \mathrm{Zn})$ and hydrocarbons,

- distribution of the pollution between dissolved and particulate fractions,

- particle characteristics: pollution loads on particles, settling velocities,

- distribution of the pollution load during the rain event.

- An evaluation of the contribution of the three sources of pollution, which are urban runoff, waste water and sewer sediments, to the pollution loads measured during a rain event at the outlet of the combined sewer.

- A study of the evolution of wet weather flow pollution between the entry and the outlet of the combined sewer.

The results obtained underline the importance of combined sewers as physical and chemical reactors. The erosion, of combined sewer sediments appeared to be the main source of particles and organic matter in wet weather flows, whereas heavy metal loads mainly originated from roof runoff, due to the corrosion of metallic roofs.

\section{D CONTEXTE ET OBJECTIFS DE}

\section{LA RECHERCHE}

De nombreuses études, menées depuis les années 70 en France et à l'étranger, ont démontré l'importance de la pollution des rejets urbains de temps de pluie et leur impact sur le milieu naturel. Elles ont abouti par ailleurs au constat d'une aggravation de la pollution des eaux pluviales dans le cas des réseaux unitaires par rapport aux réseaux séparatifs. Cette aggravation, qui ne peut s'expliquer uniquement par l'apport des eaux usées de temps sec, pourrait être due à la remise en suspension des dépôts constitués dans le réseau ou encore à une différence de qualité des eaux de ruissellement entre les zones périurbaines où se situent généralement les réseaux séparatifs et les centres villes drainés par des réseaux unitaires.

C'est dans ce contexte qu'a été mis en place à Paris, au niveau d'un bassin versant urbain situé en centre ville et drainé par un réseau d'assainissement unitaire, un dispositif

* Mme Gromaire-Mertz a reçu une lettre de félicitations du jury du prix Henri Milon. expérimental permettant le suivi de la qualité des eaux urbaines aux différents niveaux du cycle de l'eau dans la ville. Il a permis :

- la caractérisation fine de la pollution des différents types d'eaux produites par le bassin versant (eaux usées de temps sec, eaux de ruissellement, eaux pluviales unitaires),

- l'évaluation de la contribution respective des trois sources de pollution (eaux usées, eaux de ruissellement et remise en suspension des dépôts en réseau) à la pollution des eaux pluviales en réseau unitaire,

- l'étude de l'évolution de la qualité des effluents au cours de leur transfert en réseau et l'évaluation des caractéristiques des particules érodées dans le réseau par temps de pluie.

Ce travail [1] a été réalisé dans le cadre du programme de recherche "Génération et Transport de la Pollution des Rejets Urbains de Temps de Pluie en Réseau d'Assainissement Unitaire", dirigé au CEREVE (Centre d'Enseignement et de Recherche sur l'Eau, la Ville et l'Environnement) par monsieur Ghassan Chebbo et financé par le Comité Inter Agences de l'Eau, la Ville de Paris, le Conseil Régional d'Ile de France, le CST-BVRE, le LCPC et l'Ecole Nationale des 
1. Plan du bassin versant et localisation des points de mesure.

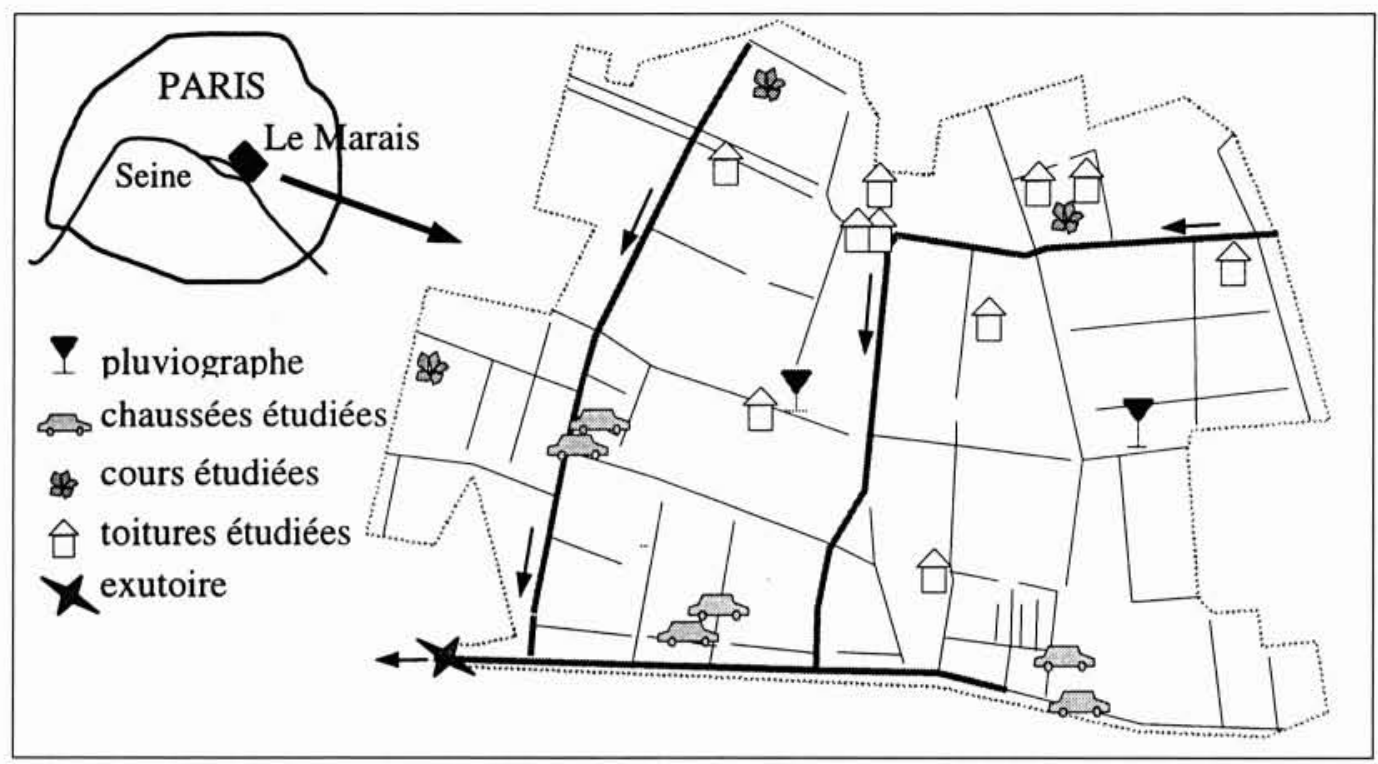

Ponts et Chaussées. Les parties concernant les métaux et les hydrocarbures ont été traitées en commun respectivement avec S. Garnaud et A. Gonzalez [2,3]. La direction de la thèse a été assurée par le professeur Michel Desbordes.

\section{II — LE DISPOSITIF EXPÉRIMENTAL}

Le bassin versant expérimental de recherche (bassin versant du Marais) qui a été mis en place dans le cadre de cette étude, se démarque par sa situation dans un centre ville dense, par son réseau de mesure très complet et par l'attention particulière qui a été portée à l'élaboration du dispositif de mesure et à l'évaluation des incertitudes.

Situé au centre de Paris, sur une partie des 3ème et 4ème arrondissements, Le Marais (Figure 1) est caractéristique d'un centre ville ancien. Il s'agit d'un quartier résidentiel avec de nombreux petits commerces et des activités du secteur tertiaire mais peu d'activités industrielles. L'habitat est dense, avec une population de 295 habitants par hectare et le taux d'imperméabilisation des surfaces est très élevé (environ 90\%).
Ce bassin versant d'une surface de 42 ha est muni d'un réseau d'assainissement unitaire, ramifié et entièrement visitable, équipé d'avaloirs non sélectifs pour le drainage des chaussées. Il comprend trois collecteurs à banquettes, d'une longueur totale de $1,8 \mathrm{~km}$, drainant une cinquantaine d'égouts élémentaires, d'une longueur totale de $5,8 \mathrm{~km}$. La pente moyenne des égouts est de $0,8 \%$ alors que celle des collecteurs est inférieure à $0,1 \%$. Le bassin versant est bien délimité et isolé des bassins alentour.

Les équipements de mesure qui ont été mis en place (Tableau 1) ont permis le suivi de la qualité des eaux pluviales depuis le ruissellement jusqu'à l'exutoire du réseau d'assainissement. L'originalité du travail effectué réside en particulier dans l'étude des différents types d'eaux urbaines. en 21 points de mesures différents ( 11 toitures, 3 cours, 6 chaussées, 1 exutoire de réseau unitaire), avec des procédures expérimentales homogènes.

Des précautions importantes ont été prises pour assurer la fiabilité des mesures. La première année de recherche a été consacrée à une description méthodique du site expérimen-

\begin{tabular}{|c|c|c|c|c|}
\hline Type d'eau & $\begin{array}{l}\text { Nombre } \\
\text { de sites }\end{array}$ & $\begin{array}{l}\text { Mesures } \\
\text { effectuées }\end{array}$ & Paramètres étudiés & Equipement \\
\hline Pluviométrie & 2 & \begin{tabular}{|c|}
$\begin{array}{c}\text { Hyétogramme de } \\
\text { pluie }\end{array}$ \\
\end{tabular} & $\varnothing$ & Pluviomètre à auget \\
\hline $\begin{array}{c}\text { Ruissellement } \\
\text { des toitures }\end{array}$ & $4+7$ & \multirow{2}{*}{$\begin{array}{l}\text { Concentration } \\
\text { moyenne }\end{array}$} & \multirow{2}{*}{$\begin{array}{l}\text { MES, MVS, DCO, } \\
\text { DBO5, Cd, Cu, Pb, } \\
\text { Zn, hydrocarbures }\end{array}$} & Fût de collecte \\
\hline $\begin{array}{l}\text { Ruissellement } \\
\text { des cours }\end{array}$ & 3 & & & $\begin{array}{c}\text { Echantillonneur automatique } \\
\text { asservi au temps + détecteur de } \\
\text { pluie }\end{array}$ \\
\hline $\begin{array}{l}\text { Ruissellement } \\
\text { des chaussées }\end{array}$ & 6 & \multirow{3}{*}{\begin{tabular}{|c|} 
Débit \\
Concentration \\
moyenne \\
Pollutogrammes
\end{tabular}} & \multirow{4}{*}{$\begin{array}{c}\text { MES, MVS, DCO, } \\
\text { DBO5, Cd, Cu, Pb, } \\
\text { Zn, hydrocarbures, } \\
\text { Vitesses de chute }\end{array}$} & $\begin{array}{l}\text { Débitmètre (chaussées : augets } \\
\text { basculants ou déversoir, }\end{array}$ \\
\hline $\begin{array}{l}\text { Unitaire temps de } \\
\text { pluie }\end{array}$ & \multirow[b]{2}{*}{1} & & & $\begin{array}{l}\text { exutoire : Ultraflux } \\
+2 \text { échantillonneurs }\end{array}$ \\
\hline $\begin{array}{l}\text { Unitaire temps } \\
\text { sec }\end{array}$ & & & & $\begin{array}{l}\text { automatiques asservis au débit } \\
\text { (monoflacon et multiflacon) }\end{array}$ \\
\hline Lavage voirie & 3 & $\begin{array}{c}\text { Débit } \\
\text { Concentration } \\
\text { moyenne }\end{array}$ & & \\
\hline
\end{tabular}

Tableau 1. - Synthèse du dispositif expérimental. 
tal, tant en surface qu'en réseau, accompagnée d'une réflexion sur le choix des points de mesure, le choix des équipements et de leurs conditions d'installation, ainsi qu'à la mise au point des protocoles d'analyse. Une attention particulière a par la suite été portée à l'évaluation de la qualité des mesures effectuées et l'estimation des incertitudes qui leur sont attachées. Cette phase préliminaire, trop souvent négligée lors de la mise en place de campagnes expérimentales, constituait une étape indispensable pour assurer la validité des mesures de pluie, de débit et de qualité, permettre leur extrapolation et leur interprétation.

La campagne de mesure a porté sur 67 événements pluvieux, pour lesquels les concentrations en MES, MVS, DCO et DBO5 ont été déterminées sur tout ou partie des sites. Une vingtaine d'événements ont été étudiés en termes de métaux et une douzaine en termes d'hydrocarbures*. Les concentrations ont été mesurées pour l'échantillon total, pour la phase dissoute et pour la phase particulaire. De plus, la répartition de la pollution au cours de l'événement pluvial et la distribution des particules par classes de vitesses de chute ont été étudiés pour une trentaine d'événements pluvieux à l'exutoire, et pour une partie de ces événements pour les eaux de ruissellement de chaussée.

\section{III — PRINCIPAUX RÉSULTATS}

\subsection{Caractéristiques des eaux de ruissellement : des éléments nouveaux}

Les résultats obtenus concernant les eaux de ruissellement ont confirmé la pollution relativement importante des eaux de ruissellement de voirie et, dans une moindre mesure, des eaux de ruissellement des cours intérieures. Ils ont montré par ailleurs que si les eaux de ruissellement de toiture sont assez peu chargées en particules et en matière organique, elles constituent en revanche une source considérable d'hydrocarbures et surtout de métaux via la corrosion des parties métalliques des toits (Figure 2). Dans le cas du bassin versant du Marais, les eaux de ruissellement de toiture qui représentent en moyenne $63 \%$ du volume ruisselé, génèrent en moyenne moins de $30 \%$ de la masse de MES, MVS, DCO et DBO5 des eaux de ruissellement mais plus de $85 \%$ du cadmium, du plomb et du zinc, et $66 \%$ du cuivre.

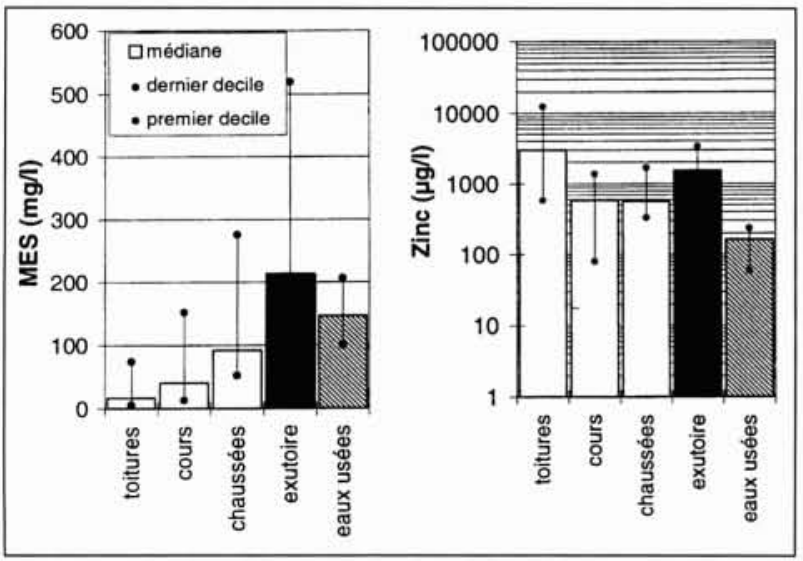

2. Ordre de grandeur des concentrations en MES et en zinc dans les différents types d'eaux urbaines (eaux de ruissellement des toitures, des cours et des chaussées, effluents de temps de pluie à l'exutoire du réseau unitaire, et eaux usées de temps sec).
L'usage de zinc, de plomb ou de cuivre pour la couverture des toitures est apparu comme hautement préjudiciable pour la qualité des eaux de ruissellement.

La caractérisation des eaux de ruissellement a fait apparaître une proportion relativement importante de pollution sous forme dissoute et des vitesses de sédimentation des particules en suspension assez faibles (vitesse de chute médiane comprise entre 0,003 et $0,52 \mathrm{~cm} / \mathrm{s}$ pour $80 \%$ des événements étudiés, voir Figure 3). Par ailleurs, pour les eaux de ruissellement provenant de petites surfaces de chaussées de 100 à $1700 \mathrm{~m}^{2}$, et bien que les concentrations mesurées soient souvent maximales en début d'événement pluvieux, on constate l'absence de phénomène de "premier flot" au sens où la majeure partie de la masse polluante serait véhiculée dans la première partie du volume écoulé (Figure 4). Ces particularités rendent assez délicate la mise en place de solutions de traitement à la parcelle des eaux de ruissellement.

\subsection{Caractéristiques des eaux unitaires de temps de pluie : confirmation ou atténuation de résultats antérieurs}

Les conclusions concernant les effluents de temps de pluie à l'exutoire du réseau unitaire ont confirmé globalement les résultats des études antérieures, en termes d'importance et de nature de la pollution :

- les particules en suspension sont le principal vecteur de pollution des eaux pluviales unitaires. A l'exutoire du Marais, 60 à $95 \%$ de la matière organique, 65 à $99 \%$ du zinc et des hydrocarbures, 90 à $99 \%$ du cadmium, du cuivre et du plomb véhiculés par temps de pluie sont liés aux particules ; - ces particules sédimentent avec des vitesses de chute supérieures à celles mesurées pour le ruissellement et nettement supérieures à celles des eaux usées de temps sec (Figure 3 ).

Ces caractéristiques permettent d'envisager un traitement par décantation des rejets unitaires de temps de pluie. Nos résultats montrent cependant qu'il convient de tempérer le postulat de bonne décantabilité des RUTP. Les vitesses de sédimentation mesurées à l'exutoire du Marais dénotent en effet d'une grande variabilité d'une pluie à une autre et sont inférieures aux valeurs citées jusqu'à présent dans la bibliographie, la différence pouvant être due aux différences de protocoles d'analyse ou au site de mesure. Une fraction non négligeable de la masse de MES décante avec difficulté : à l'exutoire du Marais, et pour $80 \%$ des événements étudiés, 17 et $39 \%$ de la masse de MES sédimente avec une vitesse inférieure à $0,002 \mathrm{~cm} / \mathrm{s}$. Il convient donc d'être extrêmement prudent lors de l'extrapolation des données de vitesses de sédimentation d'un site à un autre.

L'étude de la répartition de la pollution au cours de l'événement pluvial confirme par ailleurs l'absence de phénomène de premier flot systématique qui justifierait de ne traiter que la première fraction du volume écoulé, y compris dans le cas d'un bassin versant unitaire de petite taille tel que celui du Marais, situé très en amont du réseau et muni d'un réseau d'assainissement relativement encrassé. Les premiers $30 \%$ du volume d'un événement pluvieux véhiculent entre 20 et $60 \%$ de la masse de MES. Pour intercepter $80 \%$ de la masse de MES, il faudrait intercepter entre 54 et $83 \%$ du volume d'effluent (Figure 4).

* Les analyses de métaux et d'hydrocarbures ont été effectuées respectivement par S. Garnaud et A. Gonzalez. 


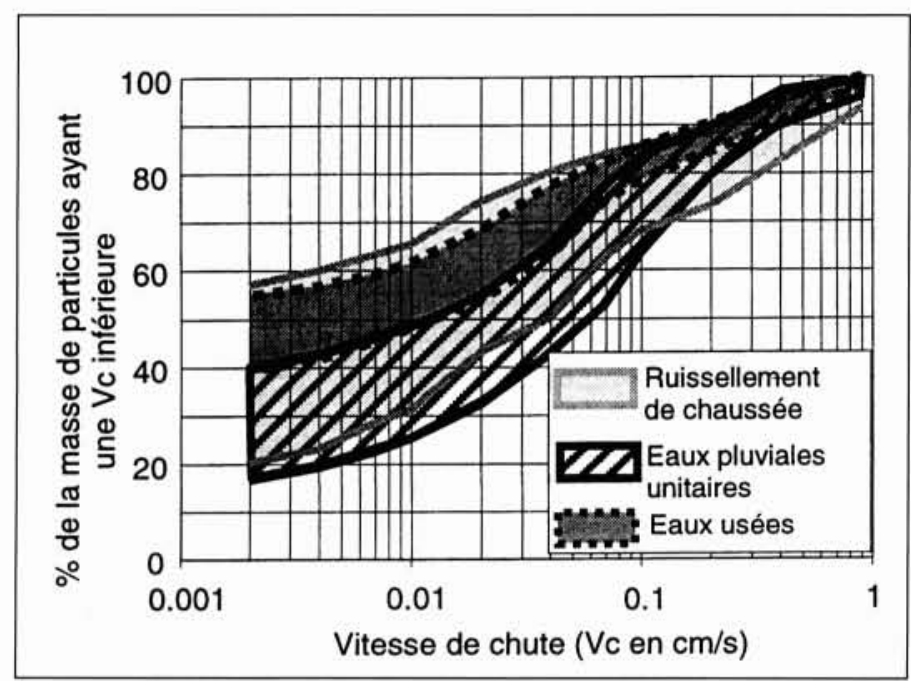

3. Distributions des vitesses de chute des particules dans les eaux usées de temps sec, les eaux de ruissellement de chaussée et les effluents de temps de pluie à l'exutoire du réseau (fuseaux correspondant à $80 \%$ des événements pluvieux).

\subsection{Le réseau unitaire : un réacteur physico- chimique au rôle fondamental}

Ce travail a mis en évidence le rôle fondamental joué par le réseau d'assainissement unitaire dans la pollution des effluents unitaires. Il démontre que le réseau n'est pas seulement un système de transport des effluents mais qu'il constitue un réacteur physique, chimique et sans doute biologique qui conditionne la qualité des effluents de temps sec et de temps de pluie.

Par temps sec, nous avons pu mettre en évidence une modification de la qualité des effluents au cours de la journée, qui s'explique, en partie du moins, par des phénomènes d'érosion et de sédimentation dans le réseau d'assainissement.

Par temps de pluie, nous avons observé une évolution de la qualité entre les eaux de ruissellement à l'entrée du réseau et les eaux pluviales à l'exutoire. On note en particulier :

- une augmentation de la proportion de polluants qui sont sous forme particulaire,

- une augmentation des concentrations en MES, MVS, DCO et DBO5 (Figure 2),

- une augmentation des vitesses de sédimentation (Figure 3),

- une augmentation de la teneur en matière organique des particules.

Le calcul du bilan des masses entrant et sortant du réseau d'assainissement du Marais au cours d'une pluie suggère

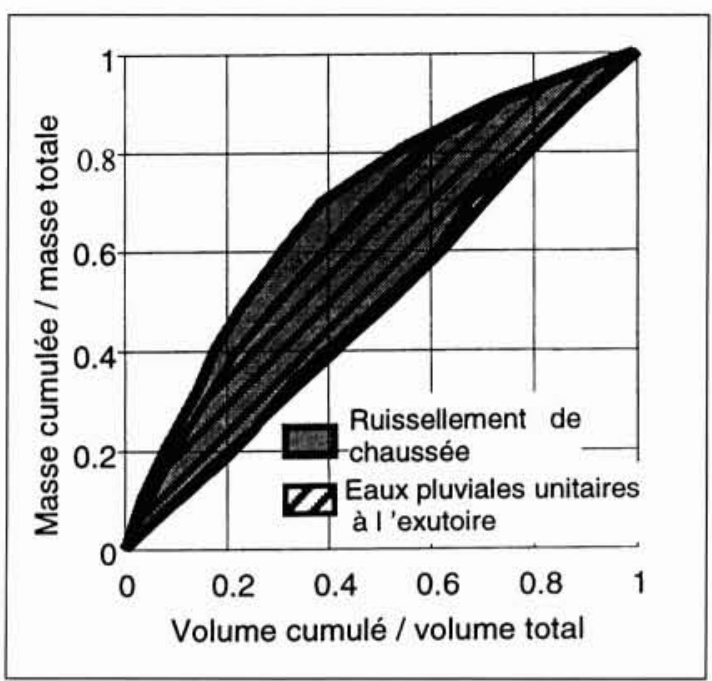

4. Fraction de la masse de MES totale véhiculée depuis le début de l'événement pluvieux en fonction de la fraction du volume total écoulé depuis le début de l'événement pluvieux (fuseaux correspondant à $80 \%$ des événements pluvieux étudiés). que l'érosion des stocks de pollution constitués dans le réseau constitue la principale source de matières en suspension et de matière organique par temps de pluie. Dans le cas du Marais, entre 40 et $70 \%$ de la pollution totale de MES, MVS, DCO et DBO5 générée au cours de l'événement pluvieux proviendrait du réseau, tandis que les eaux de ruissellement produisent moins de $30 \%$ de la masse polluante (Figure 5). D'après nos évaluations, les particules érodées dans le réseau sont fortement organiques, biodégradables, assez peu chargées en métaux et relativement décantables. Elles s'accumulent par temps sec dans le réseau et sont mobilisées progressivement au cours de l'événement pluvieux, proportionnellement à l'énergie de l'écoulement. A l'exception des vitesses de chute, leurs caractéristiques sont proches des particules en suspension dans les eaux de temps sec. Elles paraissent en revanche assez différentes de celles des particules constituant le corps des dépôts présents dans les collecteurs.

Le calcul du bilan des masses entrant et sortant du réseau d'assainissement au cours d'une pluie conduit dans le cas du cuivre à des résultats similaires à ceux obtenus pour les MES, si ce n'est que la contribution du ruissellement de toiture est plus élevée.

Les résultats sont sensiblement différents pour les trois métaux cadmium, plomb, zinc, pour lesquels la principale source de pollution, dans le cas du Marais, sont les eaux de ruissellement de toitures, par corrosion des toitures et élé-

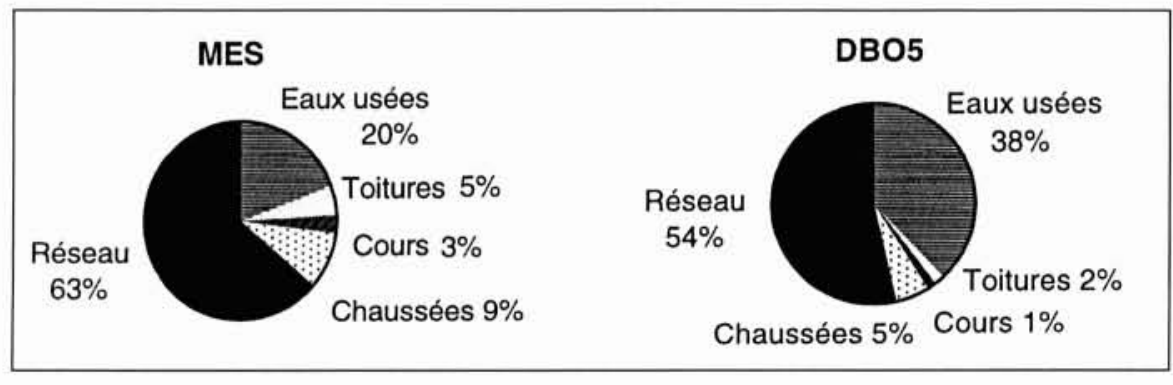

5. Contribution des différentes sources à la masse totale de MES et de DBO5 transitée à l'exutoire du bassin au cours d'une pluie (moyenne sur 31 pluies). 


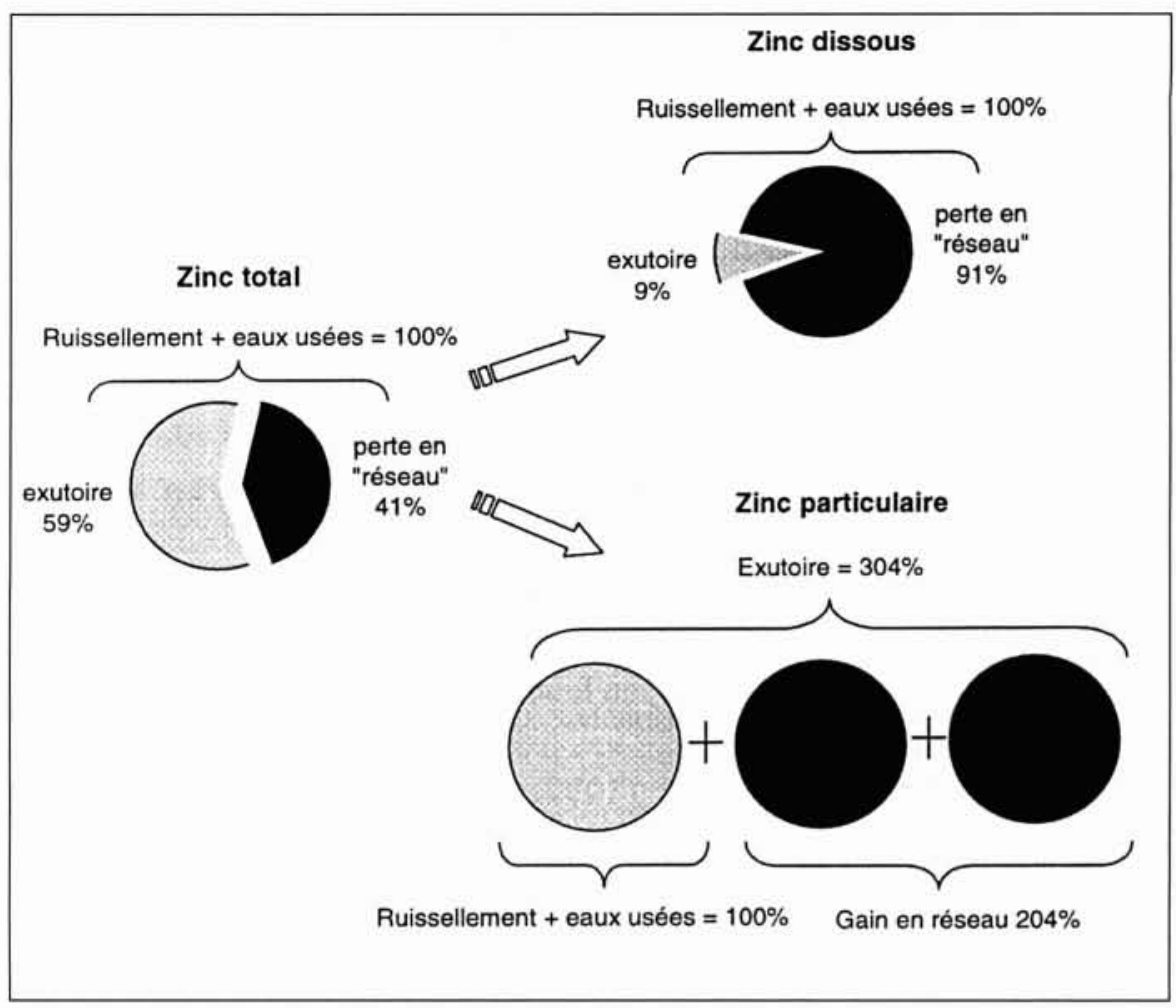

6. Illustration des pertes de zinc total et dissous et gain de zinc particulaire durant le transfert en réseau, au cours d'un événement pluvieux (valeurs moyennes sur 11 pluies).

ments de toiture métalliques. Nos bilans de masse mettent en évidence une perte de cadmium, de plomb et de zinc au cours du transport en réseau, par stockage dans le réseau. Le bilan des masses de métaux dissous et des masses de métaux particulaire entrant et sortant du réseau montre une perte massive de métaux dissous et une augmentation des masses de cadmium, cuivre et zinc particulaire. Il semble qu'il y ait une modification de la forme chimique des métaux au cours du transport en réseau, avec une fixation des métaux dissous sur les particules en suspension et sur les dépôts du réseau. Ces phénomènes sont illustrés dans le cas du zinc en Figure 6.

\section{IV $\square$ PERSPECTIVES}

Les résultats obtenus dans le cadre de cette recherche fournissent un certain nombre de pistes concernant les stratégies à adopter en vue de réduire la pollution des rejets de temps de pluie. Nous voyons que des solutions de traitement, basées sur la décantation, pourront être envisagées à l'exutoire des réseaux unitaires. Ces solutions paraissent cependant insuffisantes au vu de l'importance des volumes à traiter et il convient d'intervenir à différents niveaux, en appliquant à la fois des techniques préventives et curatives. Dans le cas des micropolluants, on s'efforcera de réduire leurs sources en ville, en intervenant notamment sur les matériaux du bâtiment. Dans le cas des matières organiques, il convient d'intervenir au niveau du réseau d'assainissement afin de réduire le stock de polluants disponible en début de pluie.

Ces résultats soulèvent également un certain nombre d'interrogations et de problématiques de recherches futures : - dans quelle mesure les résultats obtenus sont-ils extrapolables à d'autres bassins versants de taille similaire, et com- ment la qualité des effluents de temps de pluie évolue-t-elle en fonction de l'échelle spatiale considérée ?

- où se situent les stocks de polluants dans le réseau d'assainissement ? Quels sont leurs mécanismes d'accumulation et d'entraînement ?

- quel est le devenir des métaux dissous au cours du transfert dans le réseau ? Les cinétiques d'absorption sur les dépôts et les biofilms peuvent-elles expliquer les évolutions observées?

\section{RÉFÉRENCES}

[1] Gromaire-Mertz, M.-C. (1998). La pollution des eaux pluviales urbaines en réseau unitaire : caractéristiques et origines. Thèse de doctorat, Ecole Nationale des Ponts et Chaussées, 502 p. + annexes.

[2] GaRnaud, S. (1999). Transfert et évolution géochimique de la pollution métallique en bassin versant urbain. Thèse de doctorat, Ecole Nationale des Ponts et Chaussées, 396 p. + appendices.

[3] Gonzalez A. (en cours de préparation). Transport et distribution des hydrocarbures en bassin versant urbain expérimental du Marais à Paris. Thèse de doctorat, Université Paris XII.

[4] Chebbo G., Gromaire M.C., Garnaud S., Gonzalez A. (1999). The experimental urban catchment "Le Marais" in Paris. Actes de la $8^{e}$ conférence internationale Urban Storm Drainage, Sydney, Australie.

[5] Gromaire-Mertz M.C., Chebbo G. and SaAd M. (1998). Origins and characteristics of urban wet weather pollution in combined sewer systems: the experimental urban catchment "Le Marais" in Paris. Wat. Sci. Tech. 37(1), 35-43.

[6] Gromaire-Mertz M.C., Garnaud S., Gonzalez A. and Chebbo G. (1999). Characterisation of urban runoff pollution in Paris. Wat. Sci. Tech. 39(2), p. 1-8 